\title{
A Cognitive-pragmatic Approach to Puns
}

\author{
Jin Qiu \\ School of Foreign Languages, Chongqing Jiaotong University \\ No. 66 Xuefu Road, Nanan District, Chongqing 400074, China \\ E-mail: effie_qiu@sina.com
}

Received: 29-03-2013

Accepted: 14-05-2013

Published: 01-07-2013

doi:10.7575/aiac.ijalel.v.2n.4p.135

URL: http://dx.doi.org/10.7575/aiac.ijalel.v.2n.4p.135

\begin{abstract}
The present paper is intended to provide a unified account for the interpretation of puns from a cognitive-pragmatic perspective. In recent years a few scholars have delved into the processing mechanism of puns, but none of them has demonstrably elucidated the cognitive psychological foundation of puns and the development of our cognitive strategy for pun interpretation. Through careful analyses, the present paper proves that the logic of taxonomy is the cognitive psychological foundation of puns and shows that a cognitive approach incorporating the notion of the impartment and inheritance of connotation and denotation (IICD for short) can be successfully applied to the analyses of puns. Based on this notion, we propose a four-step hypothesis for the interpretation of puns. It is demonstrated that this hypothesis can effectively elucidate the interpretation of puns, and meanwhile, unfold a picture of addressees' mental progress.
\end{abstract}

Keywords: puns; the impartment and inheritance of the connotation and denotation (IICD for short); hinges;

context; cognitive mechanism

\section{Introduction}

Puns, acknowledgedly regarded as a rhetorical device and a communicative strategy, are widely employed both in literature and in daily life and have gradually received more and more scholarly attention. As a figure of speech, puns have been widely used in advertisements, jokes, riddles, literary works and the like, to make the language lively and impressive and to produce a humorous, jocular or sarcastic effect. In addition, as a universal phenomenon, puns have become an increasingly important subject in linguistic research. As Heller (1974) puts it, "The structure of the pun holds implications basic to an understanding of many psychological problems, and a knowledge of its dynamic processes offers important insights into the nature of reasoning itself." Therefore, the study of puns is believed to be of momentous and far-reaching significance.

Theories on the effectiveness of puns are literally decades old. Today, researchers who apply rhetorical, semantic or relevance-theoretic approaches to the study of puns have shifted their interests' from the various rhetorical functions (Redfern, 1984), semantic features (Guiraud, 1976; Attardo, 2004; etc.) of puns to the successful interpretation and application of it. For example, Tanaka (1992) interprets two types of puns, namely, the puns which activate two meanings without actually communicating more than one and the puns which not only activate but also communicate two meanings, within the framework of relevance theory. In addition, Mulken and her colleagues (2005), by making a quantitative study, arrive at two conclusions of puns in advertisement. Firstly, they conclude that slogans with puns are considered more amusing and are appreciated more than slogans without puns. As for the second one, they point out that slogans containing a pun with two relevant meanings are not considered more amusing than slogans containing a pun with only one relevant meaning, but the former are considered as a more felicitous choice than the latter.

Although scholars mentioned above have resorted to different approaches to the study of puns, they have undoubtedly cast light on many important issues concerning puns, such as the different types of puns, the semantic relationship between the two meanings as well as the possible interpretative processes of puns. However, two problems still remain unresolved. First of all, all the scholars so far are negligent of the cognitive psychological foundation of puns. Since this is the starting point for our cognitive mechanism, we would make endeavors to find out the essence in our following discussion. Secondly, while many studies have discussed the interpretative processes of puns in terms of relevance theory, none of them has proposed a possible solution to the working procedures of our cognitive strategy in detail.

Therefore, in what follows, we are going to investigate the above-mentioned problems in detail and try to put forward some feasible solutions from a cognitive-pragmatic perspective. To be specific, we will firstly build up a working definition and classification of puns. Then, by introducing the impartment and inheritance of the connotation and denotation (IICD) approach, we will work out the interpretative procedures of different types of puns and consequently find out a unified account for their interpretation.

\section{The Working Definition and Classification of Puns}

\subsection{The Working Definition of Puns}

Archibald A. Hill, an American scholar, proposes three key elements required for the formation of a pun, that is, double 
contexts, hinge and trigger (Fan, 1992). According to Hill, a hinge refers to a linguistic manifestation such as a homophony or a homonym, which a pun can build on. A trigger refers to the addresser's motivation to create and employ a pun. A context refers to the background against which the pun is produced or the situation in which the pun is embedded. In order to have a clear understanding of Hill's three elements, let's take the following advertisement as an example:

1) With IBM we have the power to manage the power. (an advertisement for IBM, Time, Nov. 18, 1989)

According to Hill, the three essential factors for the formation of this pun can be understood like this: The double contexts refer to people's ability to manipulate computers and an electrical switch to operate a computer. The hinge is the homonymic word "power". And the trigger is the advertisers' motivation to attract consumers to purchase their computers. But what's worth mentioning is that the homonymic word "power" should be considered as two hinges in this example as it appears twice and conveys two different meanings respectively.

Strictly speaking, we prefer the term "hinge" to the term "word" in that we believe a hinge can be in the form of not only a word, but also a phrase, a sentence, or even a paragraph. That is to say, although in most cases the hinge of a pun takes place on the word level, it is also likely to occur on other levels like phrase level or sentence level.

Hereby, we are able to establish a working definition of puns, that is, an intentional use of a hinge to activate an intended meaning, together with a literal meaning, or of two hinges with similar or identical phonological and/or graphological form to convey two different meanings; the intentionality of the employment of puns is to serve certain pragmatic purposes.

What we should specify here is that two cases are deliberately ignored in our working definition. One is the puns which are caused by the addressee's deliberate distortion and do not involve the addresser's intentionality. The other is the puns which are signaled by only one hinge but communicate more than two different meanings. We deliberately ignore these puns because it is likely too difficult for us to determine exactly the multiple intended meanings and to weigh their relative importance.

\subsection{The Classification of Puns}

The delicate classification of puns has always been a headache for scholars. Vittoz-Canuto (Attardo, 1994) puts forward a detailed classification of puns as follows: exploitation of the signifier, exploitation of the signified, homonyms, exploitation of the signified (polysemy), exploitation of the connotations, neologisms (new signified added to the preexisting ones), and others. Sherzer (1985) also holds that puns can be produced by manipulating different levels and aspects of language such as sound patterns, morphology, syntax and semantics. Besides, Vickers (1988) puts forward a very general classification of puns. According to him, puns consist of three types, that is, syllepsis, paronomasia and antanaclasis.

Up to now, we have named a few previous studies on the classifications of puns, but it seems to be insurmountable challenge to list exhaustively all of the possible classifications and establish a complete and scientific classification of puns. However, the classification of the pun is a necessity in our following studies. Therefore, we are going to roughly classify puns into two rudimental categories based on our working definition: homophonic puns and homonymic puns.

Homophonic puns refer to different words or phrases having the same or similar sound but different meanings; they are realized by taking advantage of the phonological similarity of the hinge(s). Homonymic puns are usually signaled by polysemous words or phrases and the double meanings conveyed by them are context-dependent. Examples 2) and 3) are a homophonic pun and a homonymic pun respectively.

2) Make your every hello a real good buy. (an advertisement for a telephone)

3) We must all hang together, or we shall all hang separately. (Benjamin Franklin)

As is noted above, example 2) is a homophonic pun, in which the hinge is the pronunciation of "good buy". The literal meaning of "good buy" refers to a good purchase or a good deal. However, thanks to the double contexts of a telephone conversation and a purchase situation, as well as the phonological identity between "good buy" and "goodbye", the same hinge is employed to suggest the meaning of its homophone "goodbye", that is, farewell. As for example 3), the same word "hang" which appears twice in this sentence serves as the two hinges of the pun. This pun is also produced by double contexts, namely, the current situation and the future prospect. Taking the double contexts into consideration, it is not difficult for us to draw a conclusion that the first hinge "hang" refers to being united and the second hinge "hang" refers to dying by hanging. The two identical hinges, along with the modified adverbs after them, say, "hang together" and "hang separately" form a dramatic contrast between life and death and furthermore imply the vital importance of unity.

\section{The Impartment and Inheritance of the Connotation and Denotation (IICD) Approach}

\subsection{Type Hierarchy Structure}

For cognitive linguists, categorization is an important issue, because it is something that underlies the mental processes of language comprehension. Generally speaking, categorization just refers to a mental process of classification and its products are commonly called cognitive categories (Ungerer \& Schmid, 2001). In this sense, categorization and the generalization of stereotypical relations share at least one important common point: both of them involve the mental process of classification. According to Xu (2008a, 2008b, 2008c), classification, namely taxonomy, is fundamentally constructed on people's understanding of stereotypical relations among objects and events. Consciously or 
unconsciously, people apply stereotypical relations to categorizing objects and events in the world into different conceptual systems. These taxonomic systems are the logical generalization of objects and events in the world.

Stereotypical relation can be reduced to a relation characterized by two parameters: [proximity \pm ] and [similarity \pm ], based on which, all the objects and events can be understood by means of the type hierarchy structure. That is to say, entities belonging to the same domain of interest can be sorted out to construct a space of similarity, namely, a category. Entities in the same domain of interest can be attributed to the same category in that they share some common properties of their superordinate category. Therefore, in a broader sense, any object/event in the world can be considered as an entity of a certain category; entities of the same category are the subtype hierarchy of a superordinate category. However, the status of an object/event in a hierarchy is not constant. An object/event in a supertype hierarchy can change to a sub-class type so long as it shares some common properties with other entities in the same category. To be more exact, let's take a familiar category "tree" for instance. Being in the supertype hierarchy, "tree" is the super-class of any kind of trees, such as willows, pines, oaks and the like: tree $\rightarrow$ \{willow, pine, oak, ... $\}$. Actually, it is convenient for us to highlight one or several salient properties common to different kinds of trees within the scope of our knowledge, such as "consist of a trunk, branches and leaves or needles", "provide shade", "used as fire wood", etc. However, when it is regarded as a kind of plant, together with "vine", "grass" and so on, "tree" is the sub-class type of its superordinate category, say, "plant": plant $\rightarrow\{$ tree, vine, grass, ... $\}$. As a matter of fact, these taxonomies can be more abstract or concrete. Concretely speaking, the pine, as an ordinary kind of tree, is the super-class of different kinds of pines, such as genuine pines, red pines, etc. Abstractly speaking, "plant", together with "creature", can be regarded as the sub-class type of their superordinate category: "living being". Figure 3.1 attempts to sketch these complicated taxonomies (Ungerer \& Schmid, 2001).

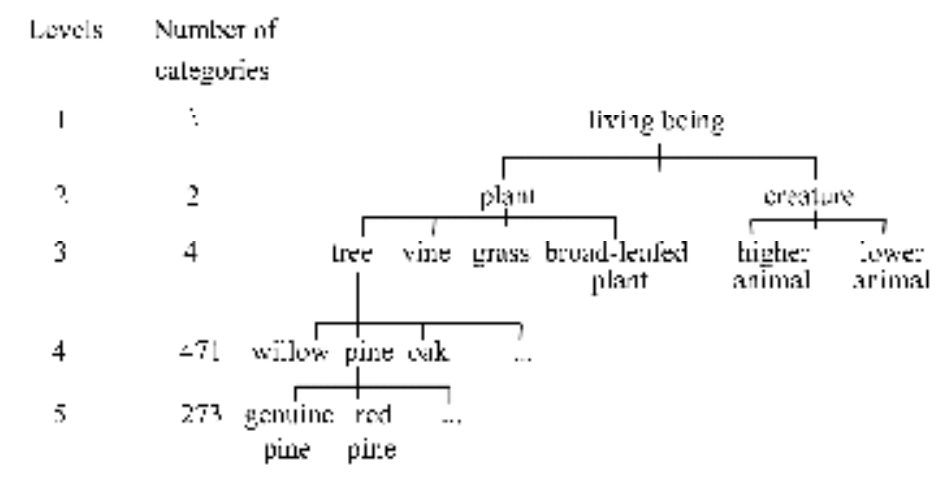

Figure 1. Tzeltal plant classification: revised

Figure 1 shows the superordinate and subordinate categories that the category "tree" involves, and the numbers of categories on different levels. As has already been indicated, plant categories are most numerous on level 4, numbering 471 in total. In contrast, the number of level 3 is exceedingly small; it contains no more than four kinds of plant. Level 2 is even smaller with only two members in total. Of the lower levels, although membership in level 5 is more restricted than in level 4, different types of pine trees have entirely been covered. Therefore, in a type hierarchy structure, the higher level an entity stands, the more abstract it will be; whereas the lower level an entity locates, the more concrete it will become. Another thing worth mentioning is that this type hierarchy structure is far from complete, and as a case stands, it will never be accomplished. Entities, categories and hierarchies are on-limits because our knowledge about the world is boundless. With the broadening of our knowledge about the real world as well as the possible world, categories and hierarchies are always on the change.

As Xu (2008a, 2008b, 2008c) classifies, type hierarchy structure consists of two types, that is, the taxonomic type hierarchy and the compositional type hierarchy. The former is the aggregate of kindred entities; while the latter is the aggregate of different parts or characteristics of an entity. These two types can respectively embody relations of proximity and/or similarity to some extent. Let's take another well-known category "flower" for example. In light of our encyclopedic knowledge, daisies, lilies, roses and the like are all members of "flower" category, which can be assorted into the taxonomic type hierarchy. In this small-scaled type hierarchy structure, the category "flower" is at the supertype hierarchy; while daisies, lilies and roses which can respectively be called as a kind of flowers stand at the subtype hierarchy. They are proximate in location within the scope of "flower" category. What's more important, all of them share the fundamental attributes of flowers, such as "being colorful", "produce seed or fruit", and so forth. In this sense, they are similar to one another. This example presents us a vivid description of the taxonomic type hierarchy, which emphasizes more on the relation of similarity than proximity. In terms of the compositional type hierarchy, it is obvious that stamens, petals, receptacles, etc. are compositional parts of a flower, among which the prominent character is that they are adjacently distributed in a flower. Therefore, the compositional type hierarchy focuses more on the relation of proximity. Type hierarchy structure and the relations among different categories will shed new light on our understanding of the world.

\subsection{Brief Introduction of the IICD Approach}

The above examples of "tree" and "flower" type hierarchy structures are by no means exceptional. They are also of great importance in the sense that they have provided an original approach to people's understanding of the world, an 
approach of logic of taxonomy. As is already explained, categories of the same level bear the relations of proximity and/or similarity; so do the adjoining hierarchies. To put it clearer, daisies and lilies, stamens and petals show relations of proximity and/or similarity on their respective levels. However, daisies and flowers as well as stamens and flowers, though belonging to different hierarchies, also embody relations of proximity and/or similarity. Figure 2 presents us a clearer picture on these relations.

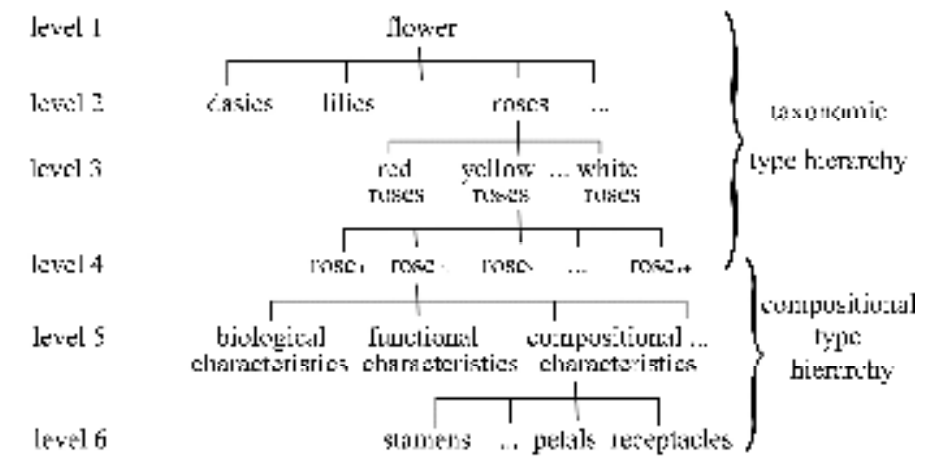

Figure 2. "Flower" type hierarchy structure

Connotation and denotation are two important terms concerning concept in logic. Connotation is the generalization of the fundamental attributes of a name; while denotation refers to a set of objects bearing all these attributes and hence labeled with the name concerned. In order to illustrate their essence, let's take "flower" for example again. As the definition suggests, "flower" is the connotation of all kinds of flowers; meanwhile, each different kind of flowers is the denotation of "flower". To be specific, in figure 2, level 1 (flower) is the connotation of all the entities in level 2 and each kind of flowers in level 2 is the denotation of "flower". Like it or not, "rose" in level 2 is also the connotation of varieties of roses in levels 3 and vice versa. As is shown in figure 2, the continuum of taxonomies from level 1 to level 4 (flower $\rightarrow$ rose $\rightarrow$ a certain kind of rose $\rightarrow$ one rose of this kind) represents the taxonomic type hierarchy. In this continuum, super-class type imparts its essential properties to its sub-class type and the entities in sub-class type inherit the basic properties from their super-class type. The sub-class type is the denotation of the super-class type. Therefore, $\mathrm{Xu}$ proposes $(2008 \mathrm{a}, 2008 \mathrm{~b})$ that the taxonomic type hierarchy reflects denotation. In other words, in the taxonomic type hierarchy, entities in sub-class type (denotation) inherit basic properties from the impartment of their super-class type (connotation). On the contrary, the compositional type hierarchy reflects connotation. As is already shown in figure 2 , level 4 to level 6 reveal the compositional type hierarchy. In level 5, biological characteristics, functional characteristics, compositional characteristics and all the other characteristics are the connotative contents of any rose in level 4; stamens, petals and all the other parts of a rose in level 6 are also the connotative contents of the compositional characteristics of a rose. Each entity in sub-class type is an indispensable part of its super-class type, which embodies connotative contents of the super-class type. Therefore, the compositional type hierarchy reflects the imparted and inherited relations of the connotation.

Generally speaking, logic of taxonomy is the foundation of our better understanding of the world. With the accumulation of our acquisition and experience, we have, consciously or unconsciously, formed type hierarchy structure of the world in mind, which can be categorized into two types, that is, the taxonomic type hierarchy and the compositional type hierarchy. The relations of proximity and/or similarity among genus-species, part-whole and partpart have made it possible to adopt the inherent relations of connotation and denotation to interpret puns. At the end of the IICD approach, one thing should be pointed out in advance, i.e., the interpretation of puns is simply concerned with the taxonomic type hierarchy.

\subsection{The Analytic Framework of the IICD Approach}

As has already been indicated in the last section, in the range of the taxonomic type hierarchy, super-class type is the connotation of its sub-class type; meanwhile, entities in sub-class type are the denotations of their super-class type. In the continuum of taxonomies, super-class type imparts its essential properties to its sub-class type and entities in subclass type inherit the basic properties from their super-class type, herein unfolding the impartment and inheritance of the connotation and denotation. According to Xu (2008c), the analytic framework of the IICD approach can be revealed as follows:

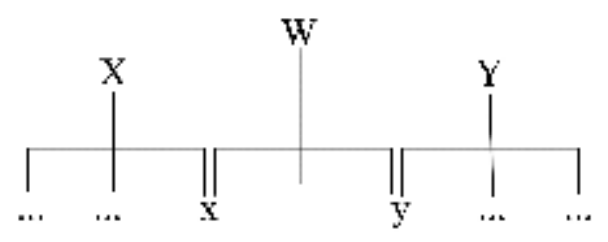

Figure 3. The analytic framework of the IICD approach

Generally speaking, entity " $\mathrm{x}$ " and entity " $y$ " respectively belong to different categories " $\mathrm{X}$ " and " $\mathrm{Y}$ ". In accordance with the analysis of the IICD approach, entity " $\mathrm{x}$ " is one of the denotations of its super-class type " $\mathrm{X}$ " and it inevitably inherits some basic properties from "X"; and entity " $y$ " likewise. However, one of the inherited basic properties of " $x$ " 
coincidentally correlates with that of " $y$ ". In this sense, entity " $x$ ” and entity " $y$ " can temporarily be ascribed to a third category "W". In this brand-new type hierarchy structure, " $x$ " and " $y$ " are the denotations of "W" and they together inherit at least one common property from "W". In light of the above mentioned principles of proximity and/or similarity, "a relational entity inherently implies the existence of another entity" (Croft \& Cruse, 2004). To put it clearer, when in certain context, the reference of " $x$ " will inherently imply the existence of " $y$ ". That is why a linguistic phenomenon, together with the context in which it is produced, is understandable, explainable and predictable.

\section{A Unified Account of Pun Interpretation}

\subsection{Proposition of a Unified Account of Pun Interpretation}

As is already indicated in section 3.2, puns are concerned with the taxonomic type hierarchy. The interpretation of puns can be approached generally in four steps. First and foremost, with the facilitation of context, communicative intention will carefully be figured out. Secondly, based on specific communicative intention, the hinge(s) of a pun will be deliberatively determined. Next, the hinge(s) will be mentally analyzed in type hierarchy structures, in which the superclass type is the connotation and entities in the sub-class type are its denotations. The last step is the constant impartment and inheritance of the connotation and denotation between the super-class and an entity in the sub-class or/and among different entities of the same class until the most appropriate understanding of the pun is approached.

In this four-step proposal, something should be noted so as to exclude misunderstandings. First, we regard communicative intention as the starting point in our pun interpretation because puns are intentionally employed to achieve certain pragmatic effects which are certainly the trigger of a pun as Hill puts it. Second, in a type hierarchy structure, the super-class imparts its basic feature to the sub-class, and the sub-class hereby inherits the basic feature from the super-class. As for homophonic puns, the basic feature is on the first place the similar or identical phonology, and then being the basic meaning of a phonologically similar word; while concerning homonymic puns, the basic feature is the basic meaning of the hinge. Third, the entities in the type hierarchy structures are inherently capable of being imparted and inherited, thanks to the relation of proximity and/or similarity among them. Last but not the least, in the course of the impartment and inheritance, context will play a crucial role in the pun understanding. In order to ensure the reliability and validity of our study, all the above steps will be applied to practice in the succeeding section.

\subsection{Application of the 4-step Proposition to the Interpretation of Different Puns}

\subsubsection{Interpretation of a Homophonic Pun}

In accordance with the classification of puns, we would like to firstly analyze a homophonic pun below:

4) Sign on a Men's Gym door, "We have courses to make grown men young and young men groan."

(Reader's Digest, June, 1981)

It is axiomatic that this sentence is coined to advertise for a men's gymnasium, which serves as the communicative intention of the pun. After the investigation, we take no pains to find that example 4) is a homophonic pun with two hinges in that the same phonology occurs twice in the advertisement, each of which conveys different meanings. Therefore, the two hinges, namely, "grown" and "groan" are effortlessly recognized. Upon our proposition, the third step of pun interpretation is the analysis of the type hierarchy structure of the hinge(s). As for the two hinges "grown" and "groan", their type hierarchy structures are to be revealed in detail in figure 4.

As is indicated below, the first hinge "grown" is composed of two denotations, that is, "the adjective form of grow" and "the past participle of grow". In the original example, the adjective form of grow is employed to modify its succeeding noun form "men", which together means men who are having full growth or being mature. The same goes for the second hinge "groan", which is the connotation of its different part of speeches, say, the noun form and the verb form. Furthermore, although different in spelling and meaning, the two hinges "grown" and "groan" can together be ascribed to a new type hierarchy structure, namely, "[grəun] $\rightarrow$ \{grown, groan\}", owing to their identity in sound.

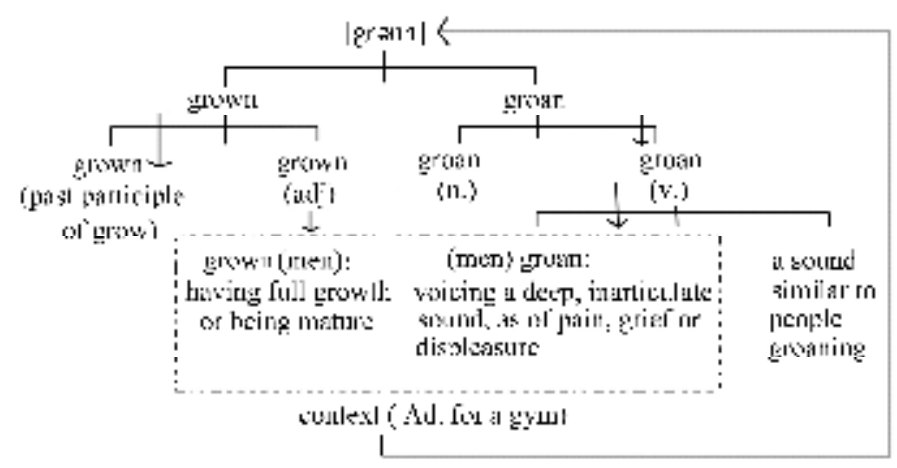

Figure 4. The comprehension of a homophonic pun with two hinges

The above type hierarchy structure makes it possible for the constant impartment and inheritance of the connotation and denotation. Based on this type hierarchy structure, "grown men" finally refers to men who are having full growth or being mature. Likewise, "men groan" implies men who are voicing a deep, inarticulate sound, as of pain, grief, or displeasure. Thereupon, context being an advertisement for a men's gymnasium, grown men are entitled to do exercises in a gymnasium; at the same time men are likely to groan in the process of doing exercises as a result of relieving 
pressure. In short, owing to the employment of an acoustic knot, these two hinges are tied together, cooperating to impress and attract more customers.

\subsubsection{Interpretation of a Homonymic Pun}

The next example of a homonymic pun is an advertisement for London Transport, which indicates "Less bread. No jam".

As is indicated, the first stage of pun processing is to find out the communicative intention of the addressers. In accordance with the context, namely, an advertisement for London Transport, we can assume that the communicative intention of this pun is probably to encourage people to take advantage of the public means of transportation, say, underground. The next step is to determine the hinge(s). This example is simply composed of four words, with no repetitive word or phrase, so it must be signaled by one hinge. In this example, "bread" and "jam" which are the stereotypical foods are naturally considered as the hinges. To be more exact, this example is actually activated by one hinge of two puns, either of which is graphologically identical.

The third stage is to analyze the hinge(s) in type hierarchy structure(s). In "bread" type hierarchy structure, "bread" comprises two connotative meanings, i. e. "a kind of food" and "old-fashioned money"; "jam" type hierarchy structure can also be finalized to "a kind of food" and "a difficult situation to move on". Stereotypically speaking, being common foods, "bread" and "jam" are initially regarded as stereotypical foods. However, these first accessed interpretations seem to be inconsistent with the context in that it is an advertisement for London Transport which was found in underground stations or trains. Therefore, these initially activated interpretations have to be discarded to give way to an appropriate context, which drives us to further our exploration. To illustrate the detailed presentation of these mental processes, figure 5 is thus provided:

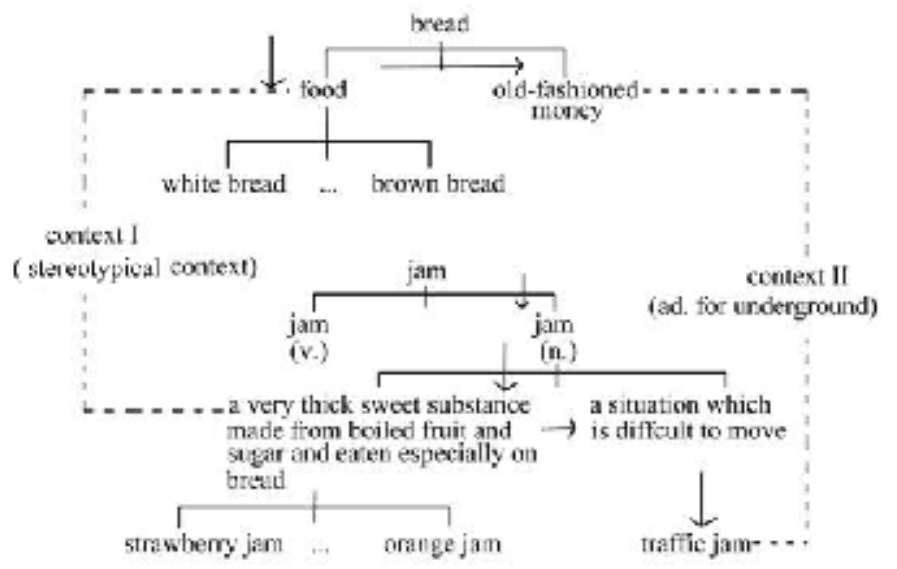

Figure 5. The IICD approach to the processing of "Less bread. No jam.”

Upon our proposition, the last stage is the constant impartment and inheritance of the connotation and denotation until the most appropriate interpretation of the pun has been reached. Figure 5 indicates the sequence of impartment and inheritance. In the taxonomic type hierarchy of "bread", the super-type "bread" firstly imparts its basic meaning to its most appropriate sub-type, say, a stereotypical kind of food. This is the first step of the impartment and the inheritance. Meanwhile, this seemingly most salient meaning is rejected because of the context. Then, on the same hierarchy, entity "food" imparts its basic feature to another denotation of "bread", say, "old-fashioned money", which finalizes the most appropriate understanding of the first hinge "bread". The sequence of the interpretation of "jam" is almost the same. As has been indicated quite clearly in figure 4.2 , we would like to omit the processes. It goes without saying that a pun should convey two meanings. In this example, the initially activated meanings of "bread" and "jam", being stereotypical foods, have given way to the less accessible, but more relevant interpretations as "money" and "traffic jam". As a result, the most appropriate interpretations of this example should be "less money and no traffic jam". Finally, one thing is still worth mentioning. Considering the intentionality of this advertisement, the addressers really want to communicate one meaning, that is, "If you travel by London Transport, it will cost you less than traveling by car, and you will suffer no traffic jams, unlike when traveling by car" (Tanaka, 1992).

\section{Conclusion}

Through careful analyses, we have tackled the two previous unsolved problems so far. Firstly, we have come to a tentative conclusion that logic of taxonomy is the cognitive psychological foundation of puns. In the process of the impartment and inheritance of the connotation and denotation, type hierarchy structure which derives from logic of taxonomy serves as the starting point of our complicated mental processes. Secondly, with the facilitation of context, chains of incessant replacement have finalized the most appropriate interpretation of puns. The interpretation of pun processing can be categorized into four steps. The first step is to determine the communicative intention of the addresser, with the help of the context. The second step aims to figure out the hinge of a pun. Next is the careful analyses of the type hierarchy structures of the hinge and the last step is the incessant impartment and inheritance of the basic feature(s) from the super-class to the sub-class until the most appropriate interpretation of the pun is approached. What's worth mentioning is that context will play a crucial role in the process of pun production and pun interpretation. 


\section{References}

Attardo, S. (1994). Linguistic theories of humor. Berlin and New York, NY: Mouton de Gruyter.

Croft, W., \& Cruse, D. A. (2004). Cognitive linguistics. Cambridge, England: Cambridge University Press.

Fan, J.C. (1992). English rhetoric appreciation. Shanghai, China: Shanghai Jiaotong University Press.

Guiraud, P. (1976). Les jeux de mots. Paris, France: Presses Universitaires de France.

Heller, L. G. (1974). Toward a general typology of the pun. Language and Style, (7), 271-282.

Mulken, V. M. et al. (2005). Puns, relevance and appreciation in advertisements. Journal of Pragmatics, (37), 707-721.

Redfern, W. (1984). Puns. Oxford, England: Basil Blackwell.

Sherzer, J. (1985). Puns and jokes. In Teun A. Van Dijk (Ed.). Handbook of discourse analysis. (Vol. 3, pp. 78-92). London, England: Academic Press Inc.

Tanaka, K. (1992). The pun in advertising: A pragmatic approach. Journal of Pragmatics, (87), 91-102.

Ungerer, F., \& Schmid, H. J. (2001). An introduction to cognitive linguistics. Beijing, China: Foreign Language Teaching and Research Press.

Vickers, B. (1988). In defense of rhetoric. Oxford, England: The Clarendon Press.

$\mathrm{Xu}$, S. H. (2008a). Why metonymy is possible - "metonymy and logic": interpreting metonymy by means of "impartment and inheritance of connotation and denotation". Journal of Shanghai Jiaotong University (Philosophy and Social Sciences), 59(1), 69-77.

$\mathrm{Xu}, \mathrm{S} . \mathrm{H}$. (2008b). Metonymy and the logic of taxonomy. Foreign Language Teaching and Research, 40(2), 93-99.

$\mathrm{Xu}, \mathrm{S} . \mathrm{H}$. (2008c). Cognitive approach to the study of rhetoric. Journal of Xi an International Studies University, 16(2), $1-5$. 